\title{
Method of magneto-elastic control of mechanic rigidity in assemblies of hydropower units
}

\author{
Vasyl' Vasyliovych Kukharchuk, V. V. Bogachuk. Valeriy Fedorovych Granyak \\ Department of theoretical electrical engineering and electrical measurements, Vinnytsia National \\ Technical University, \\ 95 Khmelnytske shose, Vinnytsia, Ukraine, 21021
}

\begin{abstract}
Paper abstract. Developed was a new method of control of mechanic rigidity in assemblies of hydropower units that allows a real-time control of technological process, and proposed was the design for a primary measuring transducer of mechanic intensity as a component of the control method proposed, with its transformation equations obtained.
\end{abstract}

Key words: control, hydropower unit, elasticity coefficient, mechanic intensity, vibration displacement, mechanic rigidity.

\section{INTRODUCTION}

Disrupted mechanical strength of hydropower units' support structure elements is a dangerous phenomenon that may result in occurrence of severe emergency situations. Therefore control of mechanic strength parameters in assemblies of hydropower units is a significant measure aimed at avoiding the breakdowns caused by their mechanic destruction that may be associated both with hazards threatening the power plant staff and with large-scale damage to equipment and, as a consequence, with major financial losses (for instance, as it was the case with Sayano-Shushenskaya hydropower plant). However, the existing methods mostly do not allow conducting direct measurements of current mechanic strength parameters in the structure during real-time equipment operation, being limited to measurement and control of indirect technical parameters, deviations of which may particularly be associated with reduction of mechanic strength (vibration displacement, acoustic characteristics etc.) [1, 2]. And since the said technical parameters, though enabling quite an effective detection of defect origination, do not ensure the opportunity of an unambiguous diagnostics of reduction in mechanic strength of hydropower units' separate key supporting assemblies [1], apparently there is the need in development of new methods for measuring the mechanic rigidity (elasticity coefficient) of support structures that would enable sufficiently effectively to detect disruptions of equipment's mechanic strength in real-time manner.

\section{SETTING THE TASK}

The main difficulty in measuring the elasticity coefficient of hydropower unit's support structures is that measuring this parameter under the classic method requires application of test reference force and measuring the absolute deformation of the material tested [3]. It is evident that performance of the described investigation on the operating equipment affected by unbalanced mechanical forces, is impossible. Besides, generation of additional external mechanic load, the influence of which was not envisaged at the stage of unit designing is undesirable, and in some cases even unacceptable. In view of the foregoing, one can conclude that, in order to measure the mechanic elasticity coefficient in the mode of real-time equipment operation the methods that provide for availability of external mechanic force are inefficient and in a number of cases unacceptable.

In terms of solution of the task set interesting is the use, as an external deformation force, of unbalanced mechanic efforts that emerge during hydropower unit operation, particularly under normal operation mode. As noted in paper [4], such efforts may be determined by various factors (such as hydroflow nonuniformity, nonideality of rotary centering etc.) and have the value that may vary at random within a particular interval. At the same time, the said efforts, the values of which, though depend to a significant degree on equipment operation mode and load, are present in all modes of its operation [4]. But, since the latter vary at random and result in vibration displacement, which as a result of random nature of mechanic efforts are also of 
random nature, the use of the said parameters for the purpose of mechanic rigidity measurement requires ensuring a high-precision measurement of mechanic intensity and vibration displacement in the mode of real-time unit operation. Hence it is evident that the main task that should be accomplished in building the systems of mechanic rigidity control in hydropower unit assemblies lies in development of methods and means allowing to ensure high-precision measurement of absolute vibration displacement and mechanic intensity in the material of hydropower unit assembly under real-time mode of equipment operation.

\section{ANALYSIS OF TASK SOLUTION WAYS}

As was shown in papers [5, 6], a promising method of measuring the absolute vibration displacement lies in measurement of absolute vibration acceleration using piezo-electric accelerometer with further analytical calculation of absolute vibration displacement using one of the algorithms proposed in the papers. Therefore, the main scientific-applied problem that has to be solved in building the method of measuring the mechanic rigidity lies in development of mechanic intensity channel that would ensure the opportunity to measure the latter under the mode of real-time hydropower unit operation without disrupting the mode of its operation.

As is known, ferromagnetic materials, including the material of hydropower unit structural elements, possess numerous various effects. These are such effects as: magnetoresistive effects, magnetooptical effects, magnetic-resonance effects, thermal-magnetic effects, magnetostrictive and magneto-elastic effects [7]. Each of the specified effects allows building different, in terms of their structure and purpose, measurement devices and transformers. While in terms of building the mechanic intensity channels, particularly promising is the use of magneto-elastic effect (Villari effect) to be observed in the material of the unit's structural elements and to enable the associate the latter with change in their instantaneous magnetic conductivity. However, a significant problem with using this effect lies in the absence of a clear mathematical model that would unambiguously describe the said dependence, without which the opportunity to use Villari effect to build mechanic intensity channels is quite limited. Hence it is evident that solution of the task to develop the method and means of measuring mechanic rigidity in hydropower unit assemblies may primarily be confined to solution of the task to develop the mathematical model of magneto-elastic effect and the design of primary measuring transducer that will based on the use of the said effect.

Let us consider the existing interpretation of the operating principle of ferromagnetic transformer (FMT), which is based on the use of magneto-elastic effect. Magneto-elastic effect, or Villari effect, is the phenomenon of change in magnetic conductivity of ferromagnetic sample with constant value of intensity of external magnetizing field when the sample is affected by external mechanic force [8].

According to [9], the magneto-elastic effect is accounted for change in domain structure, that is increase of the volume of those magnetic material domains, the energy of which is reduced under the influence of mechanic stresses. This takes place in the ferromagnetic sample, the magnetization curve of which is located in the so-called domain border shift zone, which is determined in Kondorskiy theory. But it is very hard to perform quantitative assessment of this phenomenon due to many reasons [9]. Besides, according to this theory the shift of domain border takes place with increase of the external field to the value of saturation induction. Accordingly, Villari effect will not take place in the sample affected by variable external mechanical stress, and the value of external field is a constant $H_{\text {зовн }}=$ const, which does not reflect reality $[7,10]$.

It follows from the foregoing that the need appears in a more precise interpretation of this phenomenon. It is known that magnetic moment of $\boldsymbol{M}$ atom resulting from gyromagnetic spin anomaly will not be antiparallel to total mechanic momentum. This phenomenon is very well depicted in fig.1, where the vector of resultant mechanic momentum of atom $P_{J}$ electronic shell is not in the same plain with the vector of resultant magnetic momentum of atom $M$ electronic shell, which is quite reasonably proven in paper [10]. 


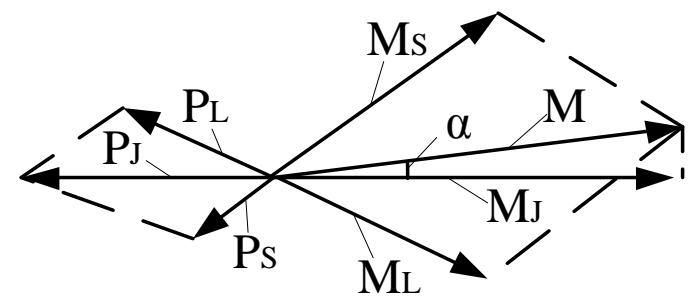

Fig. 1. Addition of magnetic and mechanic momenta of atom electronic shell

$M_{L}$ and $M_{S}$ components of magnetic momentum precess around $P_{J}$ direction. In this case the components perpendicular to $P_{J}$ momenta amount to zero on the average during rotation period, since uninterruptedly changing their direction, with total magnetic momentum $M_{J}$ of atom's electronic shell being determined by momentum components parallel to $P_{J}$, i.e.

$$
M_{J}=M_{S} \cos \left(P_{S}, P_{J}\right)+M_{L} \cos \left(P_{L}, P_{J}\right) .
$$

Thus, well founded appears to the hypothesis put forward by V.Yu. Larin, this hypothesis explaining FMT operating principle [11]: changing the vector of mechanic momenta of atoms constituting the domains of FMT material, which change results from external mechanic stress, causes change in vectors of magnetic momenta of these atoms' spins in proportion to gyromagnetic relation.

Provisions of this hypothesis fully correlate with previous investigations and conclusions made in particular by F.V. Mayorov, the founding father of the school of mechanic intensity magneto-elastic sensors, who was the first to approach the development of the said sensors from the perspective of contemporary ferromagnetism theory [12]. Specifically, he designed, investigated and showed by experiments the feasibility to use throttle measuring transducer for measurement of static and dynamic mechanic efforts. And also, based on the relationships for magneto-elastic energy worked out by N.S. Akulov [13], proposed was the analytical expression of dependence between relative change in magnetic conductivity $\mu$ and mechanic intensity of the material, which perfectly well fits in the hypothesis put forward by V.Yu. Larin:

$$
\frac{\Delta \mu}{\mu}=\frac{1}{\pi} \lambda_{0} \mu_{0} G
$$

where $\lambda_{0}$ - material's magnetostriction coefficient; $\mu$-material's magnetic conductivity in the absence of mechanic intensities; $\mu_{0}$ - magnetic stable; $\mathrm{G}$ - current mechanic intensity in the material.

According to experimental study records cited by the author, the error of proposed model was primarily explained by magneto-elastic hysteresis (up to $2.5 \%$ under static and up to $5 \%$ under dynamic operating modes), and by thermal effect (not exceeding $0.2 \%$ per $1{ }^{\circ} \mathrm{C}$ ) [12]. Given the dynamic operating mode and no fixed temperature of support elements in hydropower unit structure, one can easily conclude that proposed mathematical model may only be implemented together with temperature sensor that would allow entering a temperature adjustment into the measurement result, which significantly complicates both the very channel's design and the process of numerical processing of the measurement result. Consequently, proposed mathematical model is not completely suitable for obtaining the transformation equation of mechanic intensity sensor.

However, F.V. Mayorov has shown that, where magneto-elastic measurement transformer indicated dynamic forces that varied at up to $1 \mathrm{kHz}$ (mechanical shock mode), the delay period of magnetic conductivity change did not exceed 0.5 millisecond, which allows considering this type of measuring transducer to be actually inertia-free [12].

An alternative mathematical model describing Villari effect in magnetic material was also proposed by I.I. Ivanova in papers $[14,15]$. Specifically, the author proposed the mathematical expression that enables determination of ferromagnetic material sensitivity [15]

$$
S_{M}=\frac{2 \lambda_{s} \mu}{B_{S}^{2}},
$$

where $\lambda_{S}-$ magnetostriction coefficient at saturation; $B_{S}$ - magnetic field induction at saturation; $\mu-$ magnetic conductivity of sensor material in the absence of mechanical stresses.

Accordingly, the dependence between the relative variation in magnetic conductivity $\mu$ and the mechanic intensity of material may be shown in the form of the following equation: 


$$
\frac{\Delta \mu}{\mu}=S_{\mu} G=\frac{2 \lambda_{s} \mu}{B_{S}^{2}} G .
$$

In papers $[14,15]$ the author investigates the very transformer only, irrespective of the circuit it may be connected to. And the investigation was conducted exactly by the example of widespread electrotechnical steels (iron-silicon iron-chromium alloys) used in particular as the material of hydropower units' structural elements [16]. Based on the investigations, the author made the following conclusions:

1. Sensitivity of throttle-type magneto-elastic transformers increases with the increase in intensity of magnetic field and reaches its maximum value at the intensity that corresponds to maximum conductivity, while reducing with the increase in elastic mechanical intensity higher than particular borderline value, since magnetostriction value reduces under load.

2. Transformer's error caused by magneto-elastic hysteresis is the consequence of the two processes: mechanic hysteresis caused by elastic response that occurs under any mechanic intensities, and magnetic hysteresis resulting from specific nature of magnetizing processed.

3. Sensor operation stability will only be ensured under relatively minor loads being 6 to 7 times less than the elasticity limit.

4. With temperature variations within $\pm 10{ }^{\circ} \mathrm{C}$, magnetization current within $\pm 10 \%$ and current frequency within $\pm 0.2 \%$ the total error should not exceed $4.5 \%$.

In view of the foregoing and of the fact that operating conditions of hydropower units' structural elements do generally correspond to the requirements put forward by I.I. Ivanova in relation to magneto-elastic sensor (mechanic intensity of structural elements under normal operating mode not exceeding 1/6 to 1/7 of the elasticity limit, temperature variations in support elements of the structure being within $\pm 10{ }^{\circ} \mathrm{C}$, the magnetization current may actually be ensured with the error by amplitude $\pm 10 \%$, and by frequency - \pm $0.2 \%$ ), one can conclude that the mathematical model proposed by I.I. Ivanova is quite successfully implemented in generation of transformation equation for mechanic intensity's magneto-elastic sensor.

However the proposed expression does not give an answer to the question of spatial direction of change in material's magnetic conductivity, since, as shown in paper [13], Villari effect is of anisotropic nature and consequently depends not only on the module of mechanic intensity value, but also on the spatial direction of its vector. The answer to this question may be found based on expression (1) that allows tying the atomic (domain) magnetic momentum with the vector of mechanic efforts through angle $\alpha$, which is constant for this type of material [10]. Given (1) and the fact that the change in the material's magnetic conductivity takes place in perpendicular directions in relation to magnetic momentum vector, one can conclude that in order to fix the change in magnetic conductivity one should situate the measurement plane not perpendicularly to the vector of resultant mechanic efforts, the vibration acceleration of which to be recorded by the accelerometer, but at the angle that additionally accounts for constant $\alpha$. Such being the case eliminated is the need in analytical calculation of projection of the vector of resultant mechanical atomic momentum $P_{J}$ to the axis of resultant magnetic momentum $M_{J}$. Therefore expression (4) may be rewritten in the following form with reference to randomly chosen axis of mechanic intensity direction OX:

$$
\frac{\Delta \mu_{y+\alpha}}{\mu}=\frac{2 \lambda_{s} \mu}{B_{S(y+\alpha)}^{2}} G_{x},
$$

where " $\mathrm{x}$ " index indicates that vector projection to axis OX is taken into account, while index " $\mathrm{y}+\alpha$ " that accounted for is vector projection to the axis perpendicular to OX, as additionally adjusted for angle $\alpha$ value.

It follows from the foregoing that structure of primary measuring transducer may be implemented in the form of magneto-elastic transformer, the magnetic circuit of which is displaced at angle $\alpha$ relative to OXY coordinate axes, at the same time being a part of hydropower unit assembly (fig. 2). Such being the case, with an allowance for the specificities of magneto-elastic transformer [17] and expression (5), one can obtain the following transformation equation, that will unambiguously tie the projection of mechanic intensity to OX axis with a secondary stress of magneto-elastic transformer:

$$
U_{2}=\frac{\eta U_{1} W_{2} R_{2}}{\sqrt{R_{2}^{2}+\left(\omega \frac{W_{2}^{2}}{R_{M}}\right)^{2}}} G_{x},
$$


where $U_{1}$ - the stress on one turn of primary winding; $\eta$ - coefficient of proportionality, which depends on the steel grade and relationship between sample section and the core; $\mathrm{R}_{\mathrm{M}}$ - average resistance of magnetic flux path; $\mathrm{W}_{2}$ - number of turns of secondary winding.

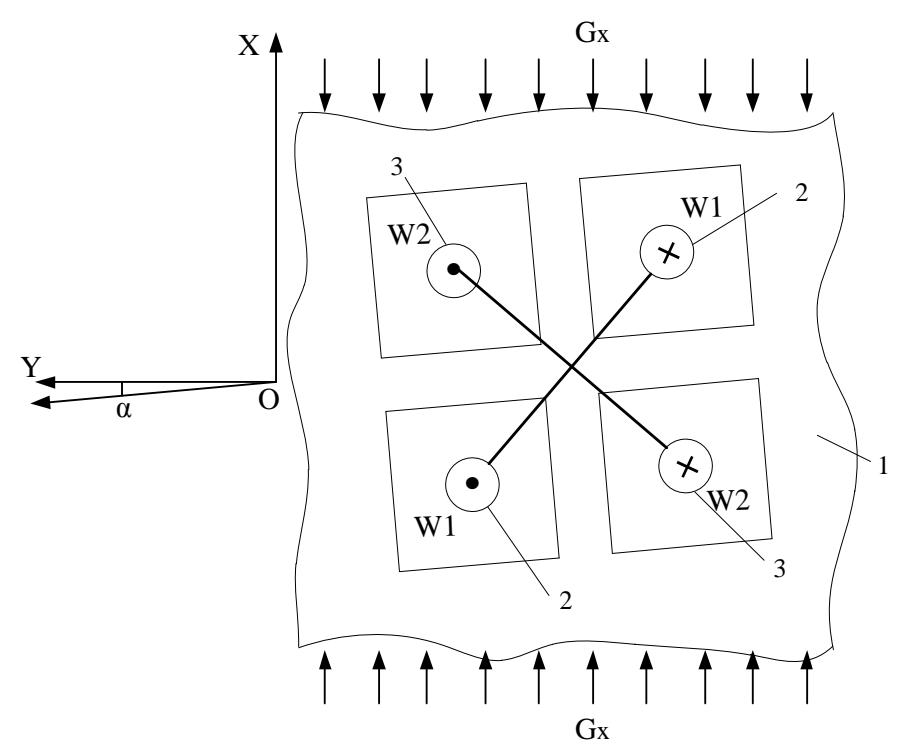

Fig. 2. Structure of magneto-elastic primary measuring transducer: 1 - material of hydropower unit assembly, 2 - primary winding, 3 - secondary winding.

In view of the foregoing one can conclude that, in presence of mechanic intensity channel and vibration displacement channel along the selected axis of mechanic intensity, the rigidity of hydropower unit structural elements may be presented in the form of the following informative parameter:

$$
\beta=\frac{\Delta x}{G_{x}},
$$

where $\Delta \mathrm{x}$ - absolute vibration displacement along the selected OX axis; $\mathrm{G}_{\mathrm{x}}$ - projection of the mechanic intensity vector in the material of hydropower unit assembly to the selected OX axis.

As it appears from (6), parameter $\beta$ will be independent from the current value of mechanic intensity in the material and vibration displacement of the latter along the selected axis, this allowing immediately to obtain information on mechanic rigidity of the structure, and therefore also to evaluate its mechanic strength. Such being the case, the operation algorithm of the device controlling the mechanic strength of hydropower unit assembly may be presented in the form shown in fig. 3 . 


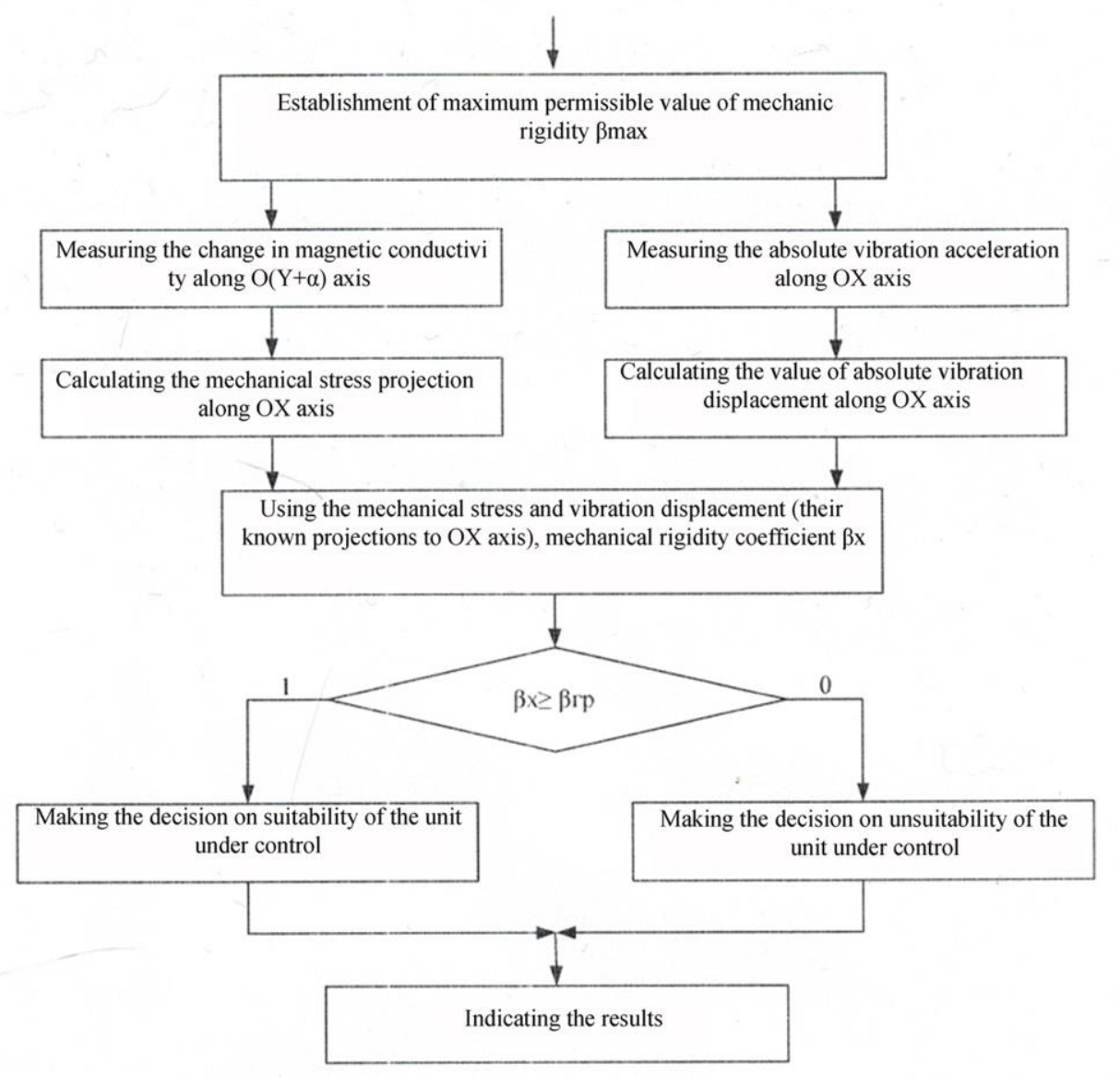

Fig. 2. Operation algorithm of the device controlling the mechanic strength of hydropower unit assembly

\section{CONCLUSIONS}

1. Obtained was the transformation equation, which unambiguously ties the value of projection module of mechanic intensity vector to OX axis with the module of vector of change in ferromagnetic material's magnetic conductivity to $\mathrm{O}(\mathrm{Y}+\alpha)$ axis, thus allowing to measure mechanic intensity in the material of hydropower unit assembly in real-time manner with a satisfactory error.

2. Proposed was the design of magneto-elastic sensor in the form of magneto-elastic transformer that allows transforming the module of mechanic intensity vector projection to OX axis by way of intermediate transformation of the latter into the vector of change in ferromagnetic material's magnetic conductivity to $\mathrm{O}(\mathrm{Y}+\alpha)$ axis, to the level of output voltage of the secondary winding of magneto-elastic transformer.

3. Proposed was the method for control of hydropower unit assembly's mechanic strength, which is based on a parallel measurement of vibration displacement and mechanic intensity projections to randomly selected OX axis, this being suitable for real-time operation without requiring availability of additional mechanical influences on hydropower unit's structural elements.

\section{REFERENCES}

1. V.V. Kukharchuk Monitoring, diagnostics and forecasting of hydropower units' vibration condition: monograph / V.V. Kukharchuk, S.Sh. Katsiv, V.G. Madiarov et al - Vinnytsia: VNTU, 2014. - 168 p.

2. A.R. Shirman Practical vibration-based diagnostics and monitoring of mechanical equipment condition / A.R. Shirman, A.B. Solovyov. - M.: Mashinostroyenie, 1996. - 276 p.

3. V.P. Babak Theoretical basis of information and measuring systems: Textbook / V.P. Babak, S.V. Babak, V.S. Yeryomenko et al; under the editorship of Corresponding Fellow of National Academy of Sciences of Ukraine V.P. Babak // - Kyiv, 2014. - 832 p. 
4. Vibrations in machinery: reference book. In 6 volumes. V. 4. Vibration processes and machines / Under the editorship of E.E. Lavendel. - Moscow, 1981. - 509 p.

5. V.V. Kukharchuk Method of analytical calculation of vibration velocity in hydropower unit acceleration mode / V.V. Kukharchuk, V.F. Granyak, Yu.G. Vedmits'ky // Visnyk Inzhenernoyi Akademii Ukrainy. - 2015. - No. 2. - p. $66-70$.

6. Yu.G. Vedmits'ky Numerical conversion of hydropower unit's vibration parameters based on firstand second-order integral vibration accelerations / Yu.G. Vedmits'ky, V.V. Kukharchuk, V.F. Granyak // Metrologiya i prilady. - 2015. - No. 5. - p. $21-27$.

7. V.Yu. Larin Theoretical substantiation of operating principle of mechanic values' ferromagnetic transformer / V.Yu. Larin // Visnyk Inzhenernoyi Akademii Ukrainy. - 2012. - No. 2 - p. 235 - 237.

8. S.V. Vonsovskiy Magnetism. Magnetic properties of dia-, para-, antiferro- and ferrimagnetics / S.V. Vonsovskiy. - Moscow: Nauka, 1971.-1032 p.

9. Physical encyclopedia / Under the editorship of A.M. Prokhorov. - Moscow: Soviet encyclopedia, 1988. $-1530 \mathrm{p}$.

10. A.A. Preobrazhenskiy Magnetic materials and elements: textbook for HEI students specializing in "Semi-conductors and dielectrics" / A.A. Preobrazhenskiy, Ye.G. Bishard. - 3th edition, revised and enlarged - Moscow: Vyshaya shkola, 1986. - 352 p.

11. V.Yu. Larin Principles of construction of ferromagnetic transformers of FoMP type / V.Yu. Larin // Scientific papers of Donetsk national technical university - 2009. - No. 176. - p. $127-135$.

12. V.F. Mayorov Magnetostrictive method and pressure-measuring instruments / V.F. Mayorov / TsAGI Papers - 1939No. - $445-$ p. $27-32$.

13. N.S. Akulov Ferromagnetism / N.S. Akulov. - Moscow-Leningrad: State publishing house for technical-theoretical literature, 1939. - $188 \mathrm{p}$.

14. I.I. Ivanova On errors of magneto-elastic transducers / I.I. Ivanova / LPI Papers - 1956. - No. 184 p. $121-127$.

15. I.I. Ivanova On calculations of magneto-elastic transducers / I.I. Ivanova / LPI Papers - 1960. - No. $8-$ p. $89-94$.

16. Power generation: history, today and future. Development of heat power industry and hydropower industry / Ye.T. Bazeyev, D.B. Bileka, Ye.P. Vasiliev et al - Kyiv: "Editors office of "Energetika" publishing house: history", 2011. $-400 \mathrm{p}$.

17. M.I. Stolbunov Ways to increase sensitivity of magneto-elastic transducers of transformer type / M.I. Stolbunov / Izmeritel'naya tekhnika - 1965 - No. 6- P. 73 - 86. 